\title{
Influence of the probe beam size on the photothermal reflectance signal
}

T. Velinov, G. Burov, K. Bransalov, J.-P. Roger* and A. Mansanares*

Sofia University, Department of Solid State Physics and Microelectronics, 5 blvd J. Bourchier, 1126 Sofia, Bulgaria

* Laboratoire d'Optique, ESPCI, 10 rue Vauquelin, 75005 Paris, France

\begin{abstract}
The influence of the probe beam size and shape on the photothermal reflectance signal is discussed. An analytical formula for the case of Gaussian power distribution is derived. The potentials of the photothermal microscope for local investigation of thermal parameters of solids and solid structures are outlined using computer simulation.
\end{abstract}

\section{Introduction}

The photothermal reflectance microscopy (PTRM) is a highly sensitive noncontact method for local investigation of opaque solids. A laser detects the small oscillations of the sample surface reflectance due to temperature and free carrier's concentration variations caused by absorbed modulated light. There are two typical applications of the PTRM - investigations of semiconductors and examinations of inhomogeneities in solids.

There exists a feasible use of the PTRM that is not exploited sufficiently although even in the first contribution on PTR such an example is given [1] - determination of thermal parameters in regions with micron dimensions. The potentialities of the PTRM for local testing of thermal parameters far exceed those of other local methods like OBD. Usually in the PTR experiments up to now the signal was thought to be proportional to the temperature of the point where the probe beam was centred i.e. the probe beam size was neglected. However in the typical PTR experiment the probe beam size is comparable to the heating beam one and to the thermal diffusion length and thus it cannot be neglected. The effect of the finite probe beam for Gaussian heating and probe beams, and homogeneous samples is treated in [2]. This paper deals with the influence of the size and the shape of the probe beam on the PTR signal. The potentials of the PT microscope for local investigation of thermal parameters of solids and solid structures are outlined using computer simulation

\section{Effect of the final beam size on the PTR signal}

It is well known that the surface temperature, when the sample is heated by a modulated laser beam is given by [3]:

(1) $T(r)=\int_{0}^{\infty} t(\lambda) h(\lambda) J_{0}(\lambda r) \lambda d \lambda$

Here $J_{0}$ is the Bessel function of order zero, $r$ is the distance to the laser beam centre, $h(\lambda)$ is the Hankel transform of the intensity source distribution and $t(\lambda)$ is a transfer function that depends on the sample 
parameters only. It is commonly assumed that the signal is proportional to the temperature of the point where the probe beam is centred. However if the two beams are focused to about the same sizes the intensity distribution of the probe beam $\mathrm{I}_{\mathrm{r}}(\mathrm{r})$ cannot be neglected and the PTR signal is proportional to the integral of the product of the temperature and the probe beam intensity. In this case the signal is:

(2) $S \propto \frac{1}{R} \frac{d R}{d T} \int T(\vec{r}) I_{r}(\vec{r}) d \vec{r}$

Here $R^{-1} \mathrm{dR} / \mathrm{dT}$ is the temperature coefficient of reflectivity. If $I_{r}$ has a Gaussian distribution with a Gaussian radius $R_{r}$ (2) can be solved analytically. Substituting (1) in (2) and changing the order of integration one can get:

(3) $S \propto \frac{1}{R} \frac{d R}{d T} e^{-\left(s / R_{r}\right)^{2}} \int_{0}^{\infty} \lambda t(\lambda) h(\lambda) d \lambda \int_{0}^{\infty} r e^{-\left(r / R_{r}\right)^{2}} I_{0}\left(2 s r / R_{r}^{2}\right) J_{0}(\lambda r) d r$

Here $s$ is the distance between the laser beam centres, $I_{0}$ is the modified Bessel function of order zero. Taking into account that :

$$
\int_{0}^{\infty} x e^{-p x^{2}} J_{0}(b x) I_{0}(c x) d x=\frac{1}{2 p} e^{\left(c^{2}-b^{2}\right) / 4 p} J_{0}(b c / 2 p)
$$

one finally obtains:

$$
S \propto \frac{1}{R} \frac{d R}{d T} \int_{0}^{\infty} \lambda J_{0}(\lambda s) h(\lambda) t(\lambda) e^{-\lambda^{2} R_{2}^{2} / 4} d \lambda
$$

This is a general solution for the case of a Gaussian probe beam. If one assumes also Gaussian distribution of the pump beam with a Gaussian radius $R_{v}(4)$ can be written as follows:

(5) $\quad S(r) \propto \frac{1}{R} \frac{d R}{d T} \int_{0}^{\infty} t(\lambda) e^{-\lambda^{2} R_{e f}^{2} / 4} J_{0}(\lambda s) \lambda d \lambda$

Here $\mathbf{R}_{\text {eff }}{ }^{2}=\mathbf{R}_{\mathbf{r}}{ }^{2}+\mathbf{R}_{\mathbf{v}}^{2}$.

The study of the influence of the probe beam shape on the PTR signal becomes important with the use of laser diodes for detection. In this case the spot has rather elliptic than circular form. We assume that in this case the probe beam has a Gaussian distribution with different radii $R_{1 r}$ and $R_{2 r}$ along the two axis. In this case Gauss-Hermit quadratures can be used:

$$
S \propto \frac{1}{R} \frac{d R}{d T} \sum_{i} \sum_{j} w_{i} w_{j} T\left(\sqrt{\left(x_{i}-s / R_{1}\right)^{2} R_{1}^{2}+y_{j}^{2} R_{2}^{2}}\right)
$$

The abcissas $\mathrm{x}$ and the weights $\mathrm{w}$ are tabulated in the literature [4]. In Fig.1 the PTR signal with an elliptic probe beam spot wirh $R_{1}=1.1 \mu \mathrm{m}$ and $R_{2}=0.9 \mu \mathrm{m}$ is presented as a function of the beam centre distance. The sample is gold, the pump beam has a Gaussian radius $R_{v}=1 \mu \mathrm{m}$ and the modulation frequency is 1 MHz. In Fig. 2 experimental results for gold at $8 \mathrm{MHz}$ are given for two perpendicular scans when a laser diode with elliptic spot is used. Details on the experimental set-up are reported elsewhere [5].

\section{Numerical PTR simulations}

In this paragraph examples will be given for thermal parameter's determination from simulated PTR signal. For that purpose the transfer function has to be known. As it was stated [6] $t(\lambda)$ for 3-D case can be found 


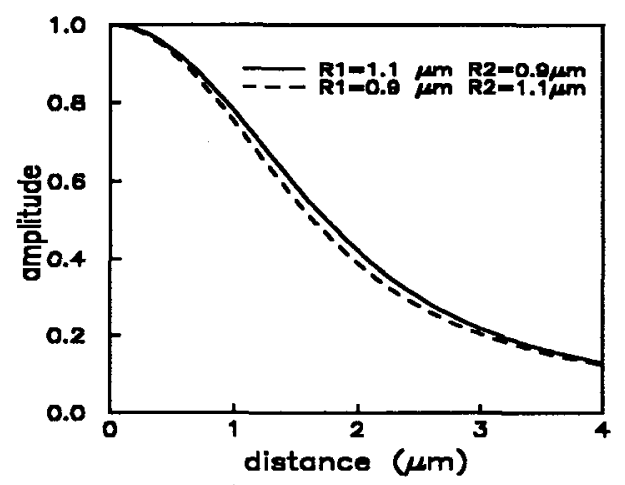

(a)

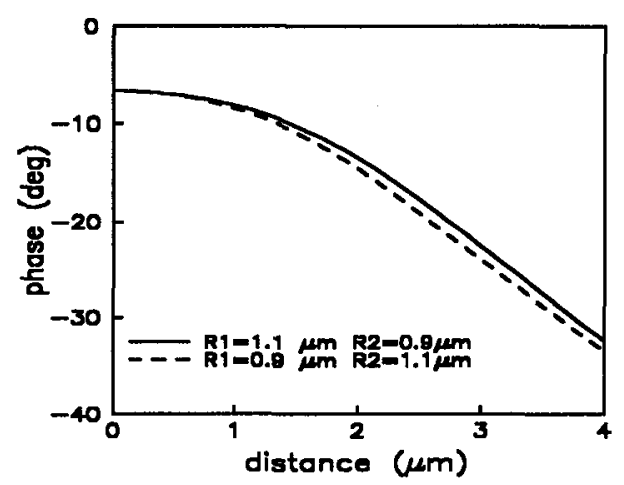

(b)

Fig.1 Amplitude (a) and phase (b) of a PTRM signal with elliptic probe beam along the two axes.

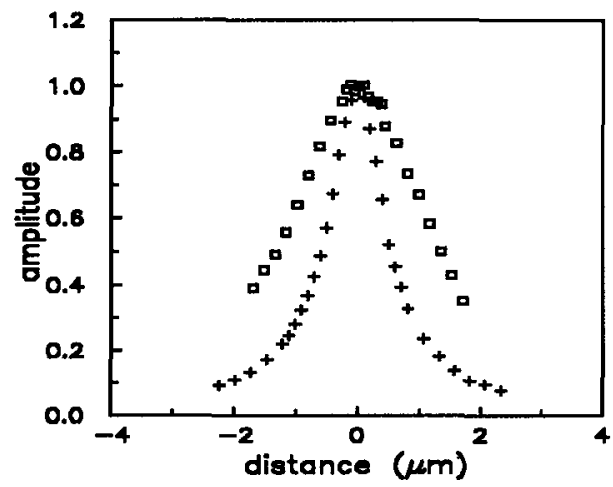

(a)

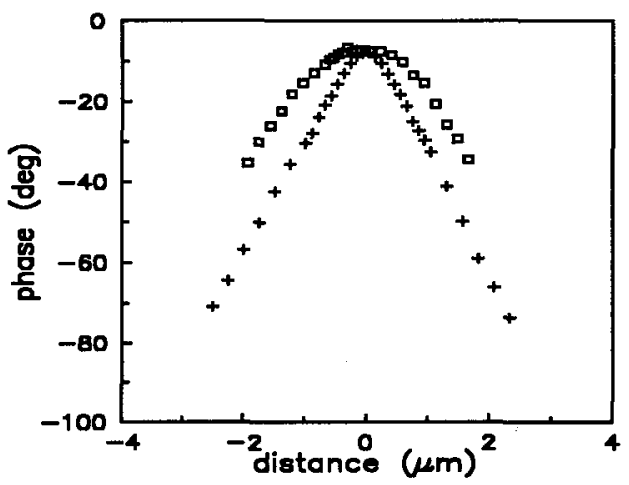

(b)

Fig.2 Experimental data at $8 \mathrm{MHZ}$ from PTRM with a laser diode for two perpendicular scans. The sample is gold: (a) amplitude, (b) phase

by replacing the thermal wave number $q^{2}=2 \mathrm{i} / \mu$ by $\mathrm{q}^{2}(\lambda)=\lambda^{2}+2 \mathrm{i} / \mu$ in the expression for the surface temperature in the 1-D case; $\mu=(\alpha / \pi \mathrm{f})^{1 / 2}$ is the thermal diffusion length and $\alpha=\mathrm{k} / \rho \mathrm{C}$ is the thermal diffusivity, $k$ is the thermal conductivity, $C$ is the specific heat, $\rho$ is the density and $f$ is the frequency of modulation, $i$ is the imaginary unit.

\subsection{Homogeneous sample}

It is not easy to determine the spot sizes of the beams when they are focused to about $1 \mu \mathrm{m}$. Moreover small displacements of the surface may cause significant changes of these sizes when objectives with large apertures are used. That is way it is useful to consider the effective radius as an unknown parameter that has to be determined from the best fit of the experimental data. The transfer function of a homogeneous opaque sample is:

(7) $\quad t(\lambda)=\frac{1}{k q(\lambda)}$

and the fitted parameters are $R_{\text {eff }}$ and $\mu$. We simulated PTRM data by evaluating (5) and adding normally distributed noise ( $3 \%$ from the amplitude and $0.3^{\circ}$ phase noise). In all cases the obtained results are practically the same as the initial values. 


\subsection{Two-layer system}

The second example deals with the potentiality of the PTRM for simultaneous determination of the thermal parameters and the thickness of a layer on a substrate. As it is known this is not possible in the one dimensional case because the surface temperature is a function of $d / \mu$. The transfer function in this case is:

(8) $\quad t(\lambda)=\frac{1}{k q(\lambda)} \frac{1+R_{b} e^{-q(\lambda) d}}{1-R_{b} e^{-q(\lambda) d}}$

Here $d$ is the layer thickness and $R_{b}$ is the thermal reflection coefficient. It is seen that (8) is not a function of the ratio $\mathrm{d} / \mu$ A procedure similar to the one described in 3.1 was used with $d, \mu$ and $R_{b}$ as fitted parameters. In the following example the starting parameters were $\alpha=0.19 \mathrm{~cm}^{2}, \mathrm{~d}=1 \mu \mathrm{m}$ and $R_{b}=0.84$ and corresponded to a Ni layer on a quartz glass. The obtained parameters are $\alpha=0.18 \mathrm{~cm}^{2}, \mathrm{~d}=0.98 \mu \mathrm{m}$ and $\mathrm{R}_{\mathrm{b}}$ $=0.85$. The effective radius has to be known in this case, otherwise the fitted parameters may be quite different from the initial values. It is worth noting that the phase and the amplitude, or the real and imaginary parts must be fitted together because different layers can give signals with almost the same real parts, but quite different imaginary parts and vice versa.

\section{Acknwoledgement}

This work is partially supported by the Bulgarian National Foundation for Scientific Investigations under contract F-71/1991.

\section{References:}

[1] Rosencwaig A., Opsal J., Smith W. and Willenborg D., Appl.Phys. Lett. 46 (1985) 1013-1015

[2] Power J. and Mandelis A. Appl.Opt. 273408 (1988)

[3] Aamodt L.C. and Murhy J.C, J. Appl. Phys. 52 (1981) 4903-4914

[4] Abramovitz M. and Stegun I, Handbook of mathematical functions (National bureau of standards 1964)

[5] Mansanares A, Velinov T, Bozoki Z, Fournier D, and Boccara A.C. to be published in J.Appl.Phys

[6] Opsal J., Rosencwaig A. and Willenborg D., Appl. Opt. 22 (1983) 3169-3176 